\title{
Ineffectiveness of intrastromal voriconazole for filamentous fungal keratitis
}

This article was published in the following Dove Press journal:

Clinical Ophthalmology

5 June 2014

Number of times this article has been viewed

\section{Masanori Niki \\ Hiroshi Eguchi \\ Yuki Hayashi \\ Tatsuro Miyamoto \\ Fumika Hotta \\ Yoshinori Mitamura}

Department of Ophthalmology, Institute of Health Biosciences, The University of Tokushima Graduate

School, Tokushima-shi, Japan
Correspondence: Hiroshi Eguchi Department of Ophthalmology, Institute of Health Biosciences, The University of Tokushima Graduate School, 3-18-15, Kuramoto-cho, Tokushima-shi, 770-8503, Japan

Tel +81886337163

Fax $+8 \mid 88$ 63I 4848

Email hiroegu0113@gmail.com
Purpose: The purpose of this study is to describe the ineffectiveness of intrastromal voriconazole injection for filamentous fungal keratitis by contrasting the effectiveness for yeast keratitis.

Methods: We examined seven fungal keratitis patients prospectively. All yeast was identified by molecular phylogenetic analyses of the chromosomal regions coding for the D1/D2 domain of the large-subunit 26S ribosomal RNA gene. All filamentous fungi were identified by the sequencing of internal transcribed spacers of the ribosomal DNA gene regions. Approximately $0.1 \mathrm{~mL}$ of voriconazole diluted with saline to $1.0 \%$ was injected with a 30 -gauge needle inserted obliquely into the three to five clear cornea sites around the abscess. All subjects were administered natamycin ointment and oral itraconazole. When needed, intravenous micafungin, voriconazole, and/or intracameral voriconazole were added. Clinical courses were observed by the slit lamp microscope. Histopathology was examined when the corneas were removed.

Results: All cases that were caused by yeast healed quickly after injections. Two cases of keratitis caused by Fusarium, and one case caused by Aspergillus, did not heal completely. In the Fusarium cases, additional antifungal medications (3.0\% topical voriconazole and intravenous injection of micafungin) were needed. After optical penetrating keratoplasty in one of the cases, fungi were found in the deep stroma of the removed cornea. In the case of Aspergillus keratitis, pathological findings also showed fungi deep in the stroma of the removed cornea and the keratitis recurred after therapeutic penetrating keratoplasty.

Conclusion: Intrastromal voriconazole injection is successful in treating yeast keratitis. However this is not the case for filamentous fungal keratitis.

Keywords: voriconazole, intrastromal injection, filamentous fungal keratitis

\section{Introduction}

Fungal keratitis is a vision-threatening infectious disease. Currently the number of effective antifungal drugs is less than those of antibacterial drugs and they are less tissue-permeable. ${ }^{1}$ Therefore fungal keratitis tends to have a worse prognosis than bacterial keratitis. ${ }^{2}$ Since filamentous fungal keratitis is more intractable than yeastrelated keratitis, ${ }^{3}$ early diagnosis and targeted treatment for filamentous fungal keratitis is the key to managing the disease. Although natamycin, the only commercially available antifungal agent for ophthalmic use, is effective in eliminating both yeast and filamentous fungi, there are some cases where its effectiveness is limited.

Voriconazole has been reported to have a broad-spectrum of antifungal properties and has been shown to be effective on ophthalmic clinical isolates, including the Fusarium and Aspergillus species. ${ }^{4}$ In recent years, some reports have shown a curative effect of intrastromal injection of antifungal drugs. ${ }^{5-9}$ Although in most of these reports, a solution of $0.05 \%$ voriconazole was effective, at our center a number of cases of filamentous keratitis recurred, despite the use of multiple intrastromal injections 
of voriconazole at concentrations of $0.05 \%$ to $1 \%$. In those cases, some causative strain showed low minimum inhibitory concentration (MIC) of voriconazole. It is important to recognize that voriconazole intrastromal injection success rate may vary depending on the underlying pathogen. Hence, we evaluate the effects of voriconazole intrastromal injection therapy for fungal keratitis and describe the ineffectiveness of the treatment for filamentous fungal keratitis by contrasting the effectiveness for yeast keratitis.

\section{Participants and methods Participants}

Seven patients (two males, five females), who were clinically diagnosed with culture-positive fungal keratitis, were enrolled in this study. All patients were consecutive fungal keratitis cases, who visited Tokushima University Hospital between January 2012 and June 2013. No patient had any antifungal medication prior to visiting our hospital, and no history of an immunocompromised state had been reported in medical interview. Their mean age was $72.3 \pm 10.8$ years (range: 60-86 years). Informed consents for the voriconazole intrastromal injection were obtained from all subjects under the approval of the Ethics Committee of Tokushima University Hospital. We excluded patients whose intraocular pressure could not be measured due to corneal perforation, cases of an involuntary eye movement, and cases where we judged that the injection under local anesthesia was impossible.

Voriconazole (1.0\%) eye drops were applied four to five times a day for at least 14 days in all cases except for Case 5. All subjects were treated with $1.0 \%$ voriconazole intrastromal injection after the diagnosis of fungal keratitis was confirmed. In the sixth case, $0.1 \%$ voriconazole was injected first and the concentration was gradually increased with each subsequent injection. In patients who had been prescribed other therapies for keratitis prior to visiting our hospital, topical antibacterial antibiotics were decreased to three or four times a day only for sterilization of the ocular surface before intrastromal injection. Additionally, topical steroids, lubricant eye drops (hyaluronic acid sodium), and the antiviral ointments were discontinued.

\section{Methods}

All isolates of fungi were obtained from the corneal scrapings. Several kinds of agar plates were inoculated with scrapings, including two Sabouraud dextrose agar (Eiken Chemical Co., LTD., Tokyo, Japan), and they were incubated at both room temperature and $37^{\circ} \mathrm{C}$. The species of yeast fungi were identified by molecular phylogenetic analyses of the chromosomal regions coding for the D1/D2 domain of the large-subunit $26 \mathrm{~S}$ ribosomal RNA gene. ${ }^{10}$ The species of filamentous fungi were identified by the sequencing of internal transcribed spacers of the ribosomal DNA gene regions. ${ }^{10}$

Voriconazole $\left(V_{F E N D}{ }^{\circledR}\right.$ for intravenous use, $200 \mathrm{mg}$, Pfizer Japan Inc., Tokyo, Japan) was diluted with saline to $1.0 \%, 0.3 \%$, or $0.1 \%$ and loaded in a $1.0 \mathrm{~mL}$ tuberculin syringe with a 30-gauge needle. Under topical anesthesia with $0.4 \%$ oxybuprocaine hydrochloride (Benoxil ophthalmic solution $0.4 \%$, Santen Pharmaceutical Co., Ltd., Osaka, Japan), the needle was inserted obliquely, with the bevel down, into the clear cornea surrounding the abscess. Approximately $0.1 \mathrm{~mL}$ voriconazole was injected at each of three injection sites in most cases. In cases where the keratitis has progressed in the whole cornea, the same dose was injected at each of five injection sites. All injections were performed in the treatment room of the outpatient clinic, unless the eye was judged to be at risk for corneal perforation due to corneal thinning. In these cases, injections were performed in the operating theater. Just after injections, all eyes were examined with a slit lamp microscope to confirm the presence/ absence of complications associated with treatment. Anterior segment optical coherence tomography (OCT) (Spectralis ${ }^{\circledR}$ OCT, Heidelberg Engineering, Heidelberg, Germany) was also performed just after the injection to confirm placement of the voriconazole solutions, as represented by stromal swelling in the mid and/or deep layers of the cornea.

\section{Results}

Four yeasts were cultured on Sabouraud plate agar. One was identified as Candida albicans and three as Candida parapsilosis. Three filamentous fungi were cultured on Sabouraud plate agar and those species were identified as an Aspergillus flavus and a Fusarium proliferatum. An additional Fusarium species was identified only by morphological character in a lacto-phenol cotton blue mount. MICs of voriconazole to all strains were determined by use of a broth dilution method according to M38-A2 of the Clinical and Laboratory Standards Institute.

Four patients diagnosed with yeast-related keratitis had complete corneal healing after administration of intrastromal injection of $1.0 \%$ voriconazole (Table 1 ). MICs of voriconazole to four Candida species were low, eg ranged less than $0.015-0.06 \mu \mathrm{g} / \mathrm{mL}$. Case 4 and 5 , where the causative fungi were A. flavus and Fusarium species respectively, were refractory to multiple $1.0 \%$ voriconazole intrastromal injections and their keratitis recurred. The MICs of voriconazole to those strains were $0.5 \mu \mathrm{g} / \mathrm{mL}$ and $4 \mu \mathrm{g} / \mathrm{mL}$, respectively. 


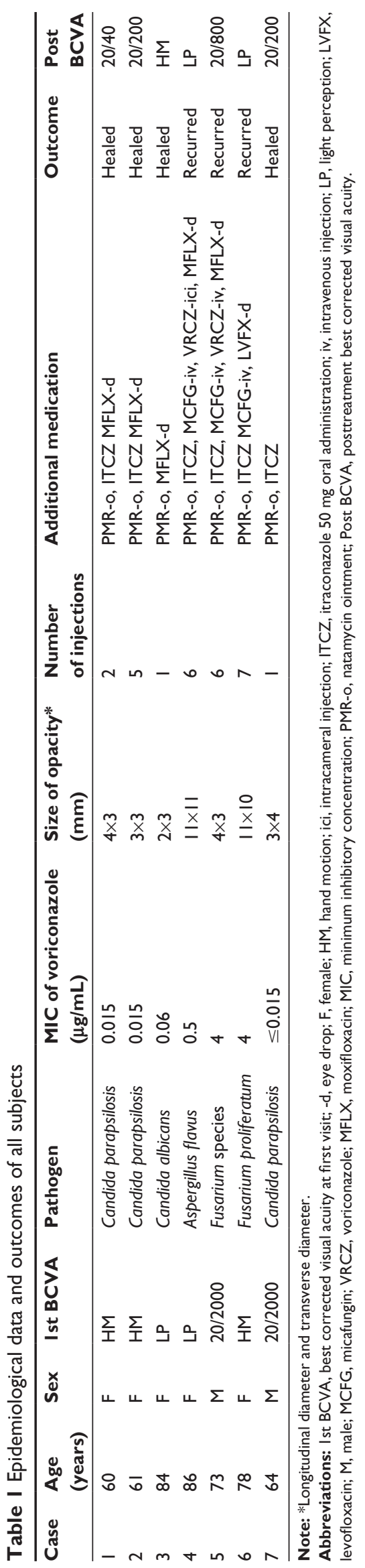

In Case 6 - the keratitis induced by F. proliferatum - we increased the concentration of voriconazole from $0.1 \%-0.3 \%$ or $1.0 \%$ because it progressed rapidly despite the additional systemic and local antifungal therapies. The MIC of voriconazole was $4 \mu \mathrm{g} / \mathrm{mL}$.

With regard to other antifungal medication, we administered supplemental antifungal medications to all cases. Topical natamycin ointment (Pimaricin ophthalmic ointment $1 \%$ Senju $^{\circledR}$, Senju Pharmaceutical Co. Ltd., Osaka, Japan) was instilled into the conjunctival sacs to all cases, $100 \mathrm{mg}$ per day of oral itraconazole capsule (Itrizole ${ }^{\circledR}$ Capsules 50, Janssen Pharmaceutical K.K., Tokyo, Japan) was given to all cases except for a mild case - Case 3 - for 2 weeks, and micafungin sodium (50 mg, Funguard ${ }^{\circledR}$, Astellas Pharma Inc., Tokyo, Japan) was injected intravenously to three persistent cases. Additionally, one subject also received an intravenous injection and an intracameral injection of voriconazole because of aphakic eye. Intrastromal injections were free from complications such as other microbial infection, corneal perforation and corneal scaring.

\section{Discussion}

In Case 4, the causative fungus was A. flavus (Figure 1), the patient did not completely recover from the keratitis after a second keratoplasty with a frozen cornea. Following the surgery, the subject was intensively treated with systemic and topical antifungal agents. Pathological examination of the first removed cornea revealed large amounts of fungi in the deep stroma adjacent to the Descemet's membrane (Figure 2) even though MIC of the causative strain of A. flavus was low. We think that either the concentration of voriconazole might have decreased faster than we expected or the solution did not reach the infectious focus. High-density OCT images

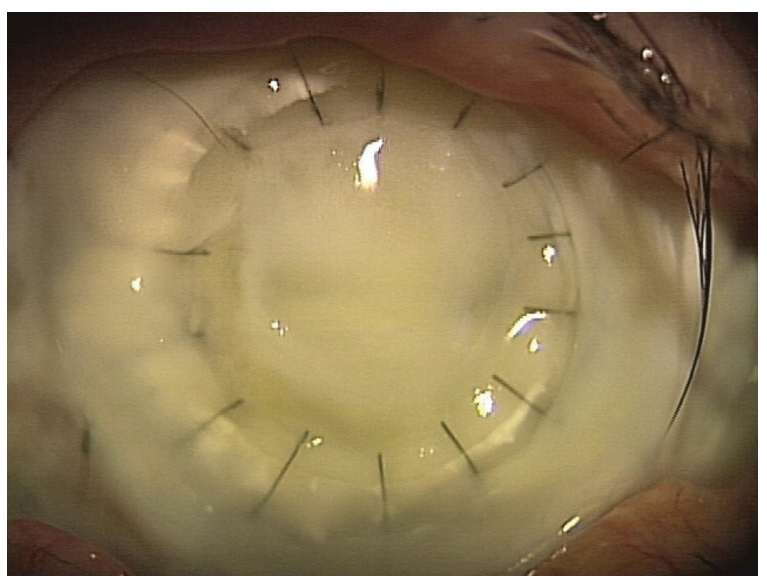

Figure I Case 4 (pre-injection). The corneal abscess caused by Aspergillus flavus has spread to most of the grafted cornea. 


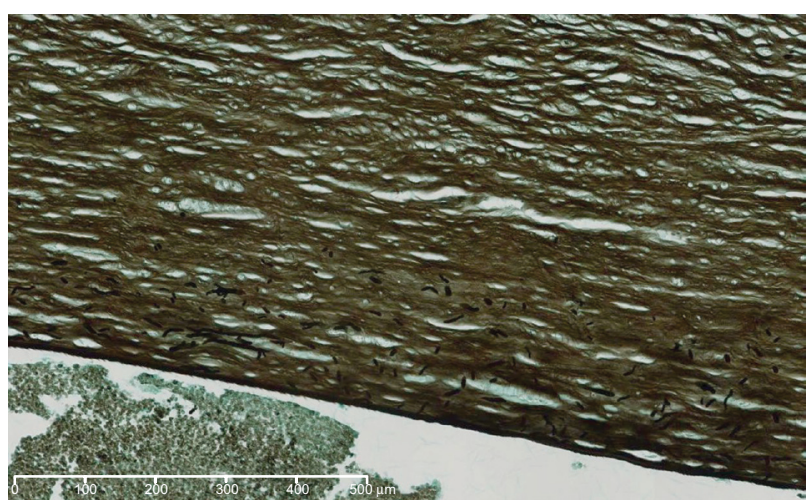

Figure 2 Case 4. Pathological findings of the removed cornea (Grocott's staining). A substantial amount of fungi are observed just above the Descemet's membrane.

suggested the presence of voriconazole at the top two-thirds or three-quarters of the cornea but it did not reach Descemet's membrane (Figure 3 ). It is important to note that the delivery of the drug is limited even by intrastromal injection.

A report ${ }^{11}$ demonstrated that the aqueous humor concentration of voriconazole declines rapidly after topical application or intracameral injection, and also demonstrated that the decline of voriconazole shows an exponential decay with a half-life of 22 minutes. Although pharmacodynamics of intrastromal voriconazole is unknown, these results suggest that a sufficient level of voriconazole in the corneal stroma may not last long after the injection. Leakage of voriconazole liquid from the stroma to the epithelial side of the cornea can also reduce the amount of voriconazole after the injection.

In Case 5, the patient suffered from a peripheral corneal perforation caused by a plant injury (normal intra ocular pressure due to an incarcerated iris) (Figure 4). The keratitis around the perforation worsened and hypopyon gradually increased, even after three intrastromal injections of $1.0 \%$ voriconazole together with hourly application of $1.0 \%$ voriconazole eye drops and nightly use of natamycin ointment. The patient was keratoplastied with the frozen cornea followed by the topical steroid for 1.5 months. Two weeks after steroid use, keratitis recurred and progressed rapidly, even with three additional $1.0 \%$ voriconazole intrastromal injections.

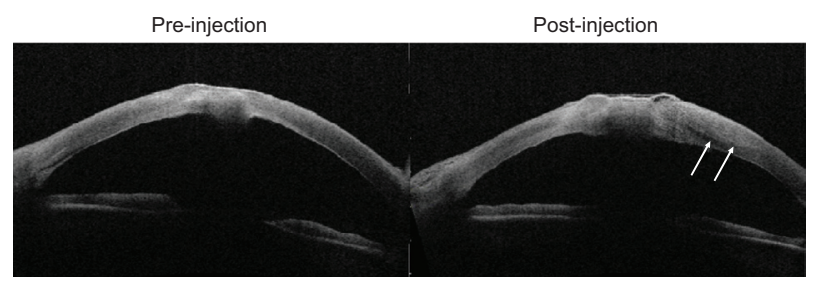

Figure 3 Optical coherence tomography images of Case I (pre- and post-injection). Notes: Intrastromal fluid location just after the injection of voriconazole can be seen in the right picture. The high-density area is limited to the outermost twothirds or three-fourths of the corneal stroma (white arrows).

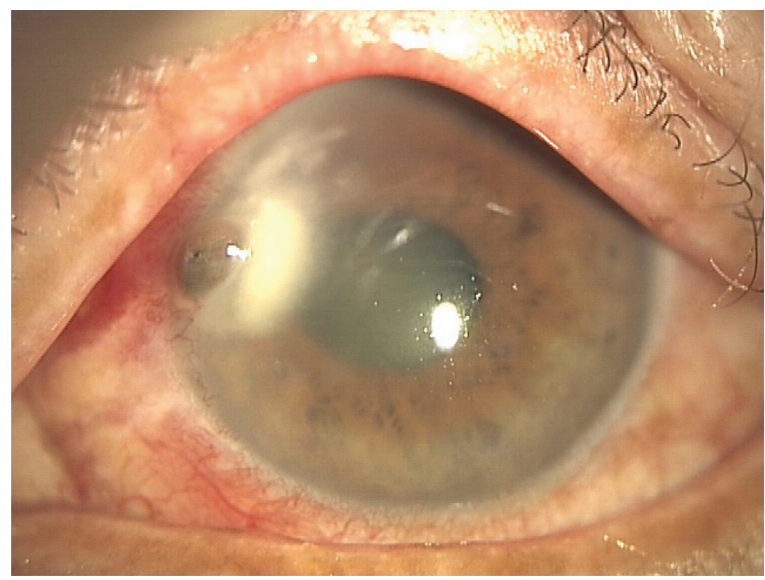

Figure 4 Case 5 (the anterior picture at the initial visit).

Note: The hyphate ulcer and the corneal perforation are observed.

Keratitis remission was finally achieved with administration of $3.0 \%$ voriconazole eye drops hourly, natamycin ointment five times daily, and intravenous injection of micafungin (100 mg/day) for 5 days. The removed cornea during optical keratoplasty revealed that hypha had infiltrated into the deep layer of the corneal stroma (Figure 5). Voriconazole delivered by intrastromal injection might not reach the infectious focus. Intrastromal injection of voriconazole was ineffective because of the rapid progress of Fusarium species keratitis ${ }^{12,13}$ that might be related to the use of topical steroid in the early postoperative stage. The Fusarium keratitis patient (Case 6) had a history of foreign body sensation during her farm work. We initiated the treatment with $0.1 \%$ voriconazole intrastromal injection together with natamycin ointment four times a day. Although the steroid was not used in this case, the keratitis was refractory to multiple injections of voriconazole. Therefore, we believe that $0.05 \%$ voriconazole is not sufficient for Fusarium-related keratitis.

Marangon et al reported that MIC of voriconazole for clinical isolates of Fusarium species showed the highest

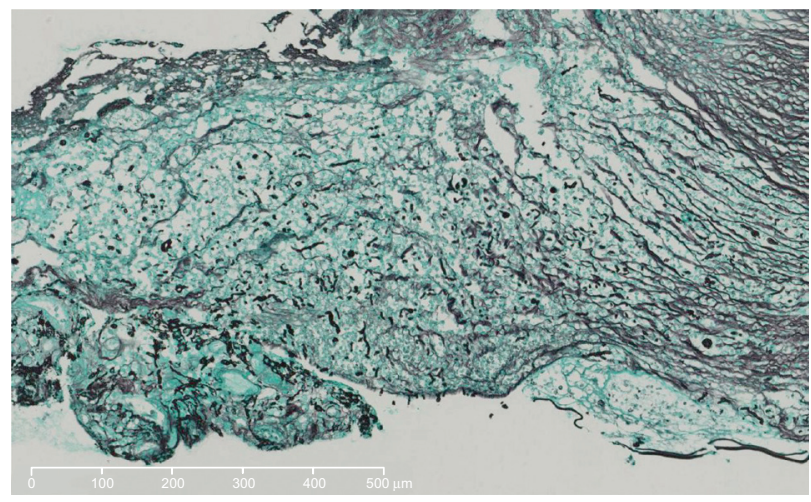

Figure 5 Case 5 (pathological findings of the removed cornea) (Grocott's staining). Note: Hyphae is observed in the deep layers of the corneal stroma. 
$(8 \mu \mathrm{g} / \mathrm{mL})$ value in all genera examined. ${ }^{4}$ The MICs of voriconazole to Fusarium species that we detected in Cases 5 and 6 were also of a comparatively high value, $4 \mu \mathrm{g} / \mathrm{mL}$. We injected between $0.3 \mathrm{~mL}$ and $0.5 \mathrm{~mL}$ of $1.0 \%$ voriconazole, which translates into $1,000 \mu \mathrm{g} / \mathrm{mL}$, at the site. This was much higher than previous reports of success with voriconazole. ${ }^{6-9}$ However, the effect of voriconazole on Fusarium keratitis is not enough for complete eradication, regardless of the concentration. In 2013, an article was published reporting that intrastromal injections of voriconazole do not offer any beneficial effect over topical $1 \%$ voriconazole therapy in a randomized clinical study. ${ }^{14} \mathrm{Cur}-$ rently we are not sure how effective voriconazole intrastromal injection is in treating Fusarium keratitis.

Although keratitis caused by the Candida species might have been successfully treated with $0.05 \%$ voriconazole intrastromal injection, the same concentration as previous reports, ${ }^{6-9}$ the optimal concentration for intrastromal injection remains to be determined. Moreover, all yeast described in this study is Candida. The response to the injection in other yeast fungi, such as Cryptococcus or Trichosporon, may differ. A recent animal study demonstrated that intracameral injection of $\geq 0.25 \%$ voriconazole could result in microstructural damage to corneal endothelial cells. ${ }^{15}$ Further animal experiments relevant to voriconazole intrastromal injection are necessary to determine optimal concentration of voriconazole with minimal toxicity to corneal endothelial cells. A further prospective clinical study with fixed treatment protocol is necessary for redeeming the small sample size and the variety of treatment protocol in this study. Then, we should compare voriconazole intrastromal injection with the new treatment option, such as topical caspofungin, as to the effectiveness of fungal keratitis. ${ }^{16}$

In conclusion, $1.0 \%$ voriconazole intrastromal injection is effective in treating fungal keratitis caused by yeast. Even using lower concentrations of the voriconazole than the present study may be effective in some cases. However, a higher concentration of voriconazole, such as $1 \%$ solution, was not consistently effective in treating advanced fungal keratitis caused by filamentous fungi, especially the Aspergillus and Fusarium species.

Clinical Ophthalmology

\section{Publish your work in this journal}

Clinical Ophthalmology is an international, peer-reviewed journal covering all subspecialties within ophthalmology. Key topics include: Optometry; Visual science; Pharmacology and drug therapy in eye diseases; Basic Sciences; Primary and Secondary eye care; Patient Safety and Quality of Care Improvements. This journal is indexed on Submit your manuscript here: http://www.dovepress.com/clinical-ophthalmology-journal

\section{Disclosure}

The authors report no conflicts of interest in this work.

\section{References}

1. Johns KJ, O'Day DM. Pharmacologic management of keratomycoses. Surv Ophthalmol. 1988;33(3):178-188.

2. Wong TY, Ng TP, Fong KS, Tan DT. Risk factors and clinical outcomes between fungal and bacterial keratitis: a comparative study. CLAO J. 1997;23(4):275-281.

3. Keay LJ, Gower EW, Iovieno A, et al. Clinical and microbiological characteristics of fungal keratitis in the United States, 2001-2007: a multicenter study. Ophthalmology. 2011;118(5):920-926.

4. Marangon FB, Miller D, Giaconi JA, Alfonso EC. In vitro investigation of voriconazole susceptibility for keratitis and endophthalmitis fungal pathogens. Am J Ophthalmol. 2004;137(5):820-825.

5. Garcia-Valenzuela E, Song CD. Intracorneal injection of amphothericin B for recurrent fungal keratitis and endophthalmitis. Arch Ophthalmol. 2005;123(12):1721-1723.

6. Prakash G, Sharma N, Goel M, Titiyal JS, Vajpayee RB. Evaluation of intrastromal injection of voriconazole as a therapeutic adjunctive for the management of deep recalcitrant fungal keratitis. Am J Ophthalmol. 2008;146(1):56-59.

7. Siatiri H, Daneshgar F, Siatiri N, Khodabande A. The effects of intrastromal voriconazole injection and topical voliconazole in the treatment of recalcitrant Fusarium keratitis. Cornea. 2011;30(8):872-875.

8. Jain V, Borse N, Shome D, Natarajan S. Recalcitrant fungal tunnel infection treated with intrastromal injection of voriconazole. Int Ophthalmol. 2010;30(6):723-725.

9. Sharma N, Agarwal P, Sinha R, Titiyal JS, Velpandian T, Vajpayee RB. Evaluation of intrastromal voriconazole injection in recalcitrant deep fungal keratitis: case series. Br J Ophthalmol. 2011;95(12): $1735-1737$.

10. Guarro J, Gené J, Stchigel AM. Developments in fungal taxonomy. Clin Microbiol Rev. 1999;12(3):454-500.

11. Shen YC, Wang MY, Wang CY, et al. Pharmacokinetics of intracameral voriconazole injection. Antimicrob Agents Chemother. 2009;53(5): 2156-2157.

12. Lin HC, Chu PH, Kuo YH, Shen SC. Clinical experience in managing Fusarium solani keratitis. Int J Clin Pract. 2005;59(5):549-554.

13. Eguchi H, Kamada Y, Kanagawa, Naito T, Shiota H. Cases of keratomycosis due to Fusarium species. Jpn J of Clin Ophthalmol. 1999;53: 609-611. Japanese.

14. Sharma N, Chacko J, Velpandian T, et al. Comparative evaluation of topical versus intrastromal voriconazole as an adjunct to natamycin in recalcitrant fungal keraitits. Ophthalmology. 2013;120(4):677-681.

15. Han SB, Yang HK, Hyon JY, SHIN YJ, Wee WR. Toxicity of voriconazole on corneal endothelial cells in an animal model. Br J Ophthalmol. 2012;96(6):905-908.

16. Hurtado-Sarrió M, Duch-Samper A, Cisneros-Lanuza A, Díaz-Llopis M, Peman-García J, Vazquez-Polo A. Successful topical application of caspofungin in the treatment of fungal keratitis refractory to voriconazole. Arch Ophthalmol. 2010;128(7):941-942.

PubMed Central and CAS, and is the official journal of The Society of Clinical Ophthalmology (SCO). The manuscript management system is completely online and includes a very quick and fair peer-review system, which is all easy to use. Visit http://www.dovepress.com/ testimonials.php to read real quotes from published authors. 